CUAD. CONTAB. / BOGOTÁ, COLOMBIA, 15 (39): 853-881 / NÚMERO ESPECIAL 2014 / 853

\title{
La contribución de los sistemas de control de gestión para el éxito empresarial
}

doi: 10.11144/Javeriana.cc15-39.cscg

\section{Nuno Miguel Delicado Teixeira}

Escuela Superior de Ciencias Empresariales del Instituto

Politécnico de Setúbal, Portugal. Doctorado en Gestión en

ISCTE, Instituto Universitário de Lisboa.

Correo electrónico: nuno.teixeira@esce.ips.pt 
Resumen El ambiente de negocios se caracteriza por la globalización, la competitividad internacional y las continuas innovaciones tecnológicas. Estas condiciones competitivas hacen el proceso de toma de decisiones más complejo. Al mismo tiempo, las empresas están luchando con la escasez de recursos y con la constante necesidad de tomar decisiones informadas sobre el uso de capital. Así, la información se ha vuelto esencial y los sistemas de control de gestión que miden la eficacia y eficiencia de las decisiones se han convertido en puntos de apoyo al desarrollo sostenible de las empresas.

Esta investigación tiene como objetivo evidenciar la contribución de los sistemas de control de gestión al éxito del negocio; se utilizó la metodología de estudio de caso en una empresa portuguesa del sector de las tecnologías de la información. Su elección se debe al hecho de que en 5 años (de 2008 a 2013) ha incrementado el volumen de negocio de 10 millones a 60 millones de euros, por lo que hoy es uno de los 20 mayores grupos portugueses de ese sector y un caso de éxito mundial. Los resultados de la investigación mostraron que el sistema de control de gestión permitió a la empresa supervisar la evolución del negocio, involucrar los centros de responsabilidad en el logro de los objetivos estratégicos y actuar oportunamente sobre los desvíos en la actividad, lo que contribuye mucho a su éxito.

Palabra clave control de gestión; tecnología de información; estudio de caso; desempeño

\section{Código JEL M 41}

\section{The Contribution of Management Control Systems to Corporate Success}

\footnotetext{
Abstract Business environment is characterized by globalization, international competitiveness and continuous technological innovation. These competitive conditions make the decision-taking process more complex. At the same time, companies are struggling with resource scarcity and the constant need to take informed decisions on the
}

use of capital. In this way, information has become essential and the management control systems that measure decision efficacy and efficiency have become a support point for the sustainable development of companies. The objective of this research is to demonstrate the contribution of the management control systems to the success of a business. We used the case study methodology for the case of a Portuguese company in the Information Technologies sector. This choice is based on the fact that in a 5-year period (2008 to 2013) this company increased its turnover from 10 million to 60 million euros, making it one of the biggest 20 Portuguese groups in the sector and a case of global success. The results of the research showed that the management control system allowed the company to supervise the evolution of the business, involved the responsibility centers in the achievement of strategic objectives, and act on a timely basis on the deviations from the activity, all of which greatly contributes to its success.

Keywords management control; information technologies; case study; performance

\section{Contribuição dos sistemas de controlo de gestão para o sucesso empresarial}

Resumo O ambiente de negócio caracteriza-se pela globalização, a competitividade internacional e continuas inovações tecnológicas. Essas condições competitivas tornam mais complexo o processo de tomada de decisões. Ao mesmo tempo, as empresas estão a lutar com a escassez de recursos e com a constante necessidade de tomar decisões informadas sobre o uso de capital. Assim, a informação tornou-se essencial e os sistemas de controlo de gestão que medem a eficácia e eficiência das decisões mudaram para pontos de apoio ao desenvolvimento sustentável das empresas. Esta pesquisa tem como objetivo evidenciar a contribuição dos sistemas de controlo de gestão para o sucesso do negócio; foi utilizada a metodologia de estudo de caso em uma empresa portuguesa do sector das tecnologias da informação. Sua escolha deve-se ao fato de que em 5 anos 
(de 2008 a 2013) o volume de negócio incrementou de 10 milhões para 60 milhões de euros, pelo que hoje é um dos 20 maiores grupos portugueses desse setor e um caso de sucesso mundial. Os resultados da pesquisa mostraram que o sistema de controlo de gestão permitiu à empresa monitorar a evolução do negócio, envolver os centros de responsabilidade na consecução dos objetivos estratégicos e reagir oportunamente sobre os desvios na atividade, o que contribui demais para o seu sucesso.

Palavras-chave controlo de gestão; tecnologia de informação; estudo de caso; desempenho

\section{Introducción}

El ambiente de negocios se caracteriza por la globalización, la competitividad internacional y las continuas innovaciones tecnológicas. Estas condiciones competitivas hacen el proceso de toma de decisiones más complejo.

Al mismo tiempo, las empresas están luchando con la escasez de recursos y con la constante necesidad de tomar decisiones informadas sobre el uso de capital. Así, la información se ha vuelto esencial y los sistemas de control de gestión que miden la eficacia y eficiencia de las decisiones, se han convertido en puntos de apoyo al desarrollo sostenible de las empresas.

En este sentido, el presente trabajo de investigación tiene como objetivo evidenciar la contribución de los sistemas de control de gestión al éxito del negocio; se utilizó la metodología de estudio de caso en una empresa portuguesa del sector de las tecnologías de la información.

La elección de la empresa se debe al hecho de que en cinco años (de 2008 a 2013) ha aumentado el volumen de negocio de 10 millones para 60 millones de euros, por lo que hoy es uno de los 20 mayores grupos portugueses en el sector de tecnologías de la información y un caso de éxito mundial. Para el trabajo de investigación en curso, vamos a nombrar la empresa como Tecnológica S.A.

El trabajo de investigación desarrolla la siguiente estructura:

- Marco teórico que aborda los diferentes instrumentos de control de gestión.

- El estudio de caso, que se divide en las siguientes secciones:

- La presentación de la empresa

- Sus etapas de desarrollo

- La planeación y el control

- La definición de los centros de responsabilidad

- La evaluación de los centros de responsabilidad

- Los precios internos de transferencia utilizados

- El diálogo como un factor crítico de éxito empresarial

\section{Metodología}

Dado que el objetivo principal de este trabajo es estudiar la contribución de las herramientas de control de gestión al éxito del negocio, lo cual requiere un conocimiento profundo del funcionamiento y de los procedimientos de las organizaciones, se eligió la metodología de investigación estudio de caso.

Para Cidália Araújo, Emília M. F. Pinto, José Lopes, Luís Nogueira y Ricardo Pinto (2008), el estudio de caso es un enfoque de investigación muy apropiado cuando se trata de comprender, 
explorar o describir acontecimientos y contextos complejos, que incluyen varios factores simultáneamente.

Robert K. Yin (2009) afirma que la metodología del estudio de caso es muy importante, porque la información privilegiada de la unidad de análisis, adecuadamente triangulada con diferentes fuentes de datos, puede revelar posibles respuestas a las preguntas que se pretende encontrar con evidencia empírica.

La investigación se llevó a cabo entre 2010 y 2012 y surgió con la elaboración de servicios de consultoría cuyo objetivo era optimizar el sistema de control de gestión implantado. Por eso, la recolección de datos se basó en tres fuentes principales de información:

\section{A. Observación directa}

- Visitas a la empresa durante la preparación del presupuesto anual, con el fin de comprobar cómo se maneja la planeación.

- Presencia en el evento Kick-off, que se celebra al principio del año, cuyo objetivo es promover los objetivos y estrategias de la empresa y los diversos planos de las distintas unidades de negocio.

- Presencia en algunas reuniones mensuales que se realizan de forma individual entre el director financiero y los gestores de unidades de negocio, para discutir los resultados del período y los criterios utilizados para la asignación de los costos indirectos.

- Presencia en algunas reuniones mensuales de los directores de unidades de negocio, en las que se presentan y sustancian los resultados de cada unidad.

\section{B. Entrevistas}

- Con la administración, para conocer el proceso de planeación estratégica y el funcionamiento del sistema de control de gestión.

- Con el director de finanzas, para identificar el sistema de información y los procedimientos implementados para el control de la gestión.

- Con un gestor de unidad de negocio, para conocer el poder de decisión sobre el área de operación y sobre los recursos disponibles que cada gestor operacional disfruta, $y$ qué opinión tiene sobre el sistema de control de gestión implementado.

C. Documentos internos

- Plan estratégico

- Informes y cuentas

- Procedimientos y normas del sistema de control de gestión

La elección de estas fuentes de información buscaba dar la oportunidad de realizar su triangulación entre diferentes perspectivas y opiniones, para minimizar la subjetividad en la interpretación de los datos recogidos (Stake, 1995).

En cuanto a la estructura del trabajo, tuvo como referencia los instrumentos de control de gestión abordados por Hugues Jordan, João Carvalho das Neves y José Azevedo Rodrigues (2008): de pilotaje, de orientación del comportamiento y de diálogo.

\section{Desarrollo}

\subsection{Instrumentos de control de gestión}

"El control de gestión es un conjunto de instrumentos que motivan a los responsables descen- 
tralizados para lograr los objetivos estratégicos de la empresa, privilegiando la acción y la toma de decisiones en forma oportuna y favoreciendo la delegación de autoridad y la responsabilización" (Jordan, Neves \& Rodrigues, 2008, p. 21).

De acuerdo con la definición anterior, el control de gestión se apoya en un conjunto de instrumentos que abordan los siguientes objetivos:

- Motivar y comprometer los gestores operativos en los objetivos estratégicos de la empresa.

- Asegurar la toma de decisiones en tiempo útil con base en datos estadísticos fidedignos.

Para lograr estos objetivos, los modelos de control de gestión observan un conjunto de principios que representan las reglas fundamentales de su acción (Jordan, Neves \& Rodrigues, 2008):

- Los instrumentos no solo incluyen datos financieros. Esto se debe a que las empresas tienen objetivos de diferentes áreas y, por esto, cuando se mide el alcance de elementos como la calidad, la productividad y la innovación, tienen que recurrir a indicadores no financieros que son fundamentales en el análisis de su desempeño.

- Pretenden esencialmente la integración de los gestores en la estrategia. Por eso, la descentralización y la delegación de autoridad son esenciales en la difusión y la aceptación de la estrategia a implementar. De este modo, se busca que los gestores puedan tener el poder de toma de decisiones sobre los recursos que disfrutan, para atender las necesidades locales y contribuir a los objetivos generales.
- La decisión y la acción temprana son elementos clave del control de gestión. Por eso, la disponibilidad de datos, incluso los estimados, en la fecha prevista, es más importante que los datos exactos en plazos dilatados que no le sirven al gestor, porque pierden toda su utilidad.

- Encuadran los resultados en la historia y los valores pronosticados, lo que permite una vista completa de la evolución del negocio.

- Actúa sobre el personal y sobre su desempeño y, como tal, su aceptación es importante, porque representa un contrato entre la empresa y el gestor, en el que se especifican las sanciones y recompensas.

- Los principales actores son los gestores operativos, pues una vez que tratan de los problemas cotidianos de la empresa y del mercado, obtienen un conocimiento tan valioso que debe estar alineado con la estrategia definida.

Por eso, el control de gestión hace operacional la planeación estratégica. Mientras la planeación estratégica tiene qué ver con las grandes líneas de desarrollo de la organización en el mediano y largo plazo, para determinar directrices y políticas de gestión en horizontes de entre 3 y 5 años, el control de gestión se concentra en el corto plazo, sobre todo en los períodos anuales para definir los programas, los presupuestos y monitorizar el desempeño y el logro de los objetivos intermedios (Anthony \& Govindarajan, 2007).

Son instrumentos que se complementan entre sí. Por eso, es vital que coexistan y estén correctamente alineados. Dado que no es po- 
sible alcanzar los objetivos en el mediano y largo plazo sin un adecuado seguimiento de corto plazo, también es impensable definir planes de acción para períodos anuales sin tener una idea coherente de qué desea ser la empresa en tres años.

Pero la complementariedad no se limita a la acción de cada horizonte. En la planeación estratégica, se debe definir el camino a seguir, pues este representa la mejor adaptación posible de la empresa al medio ambiente. Normalmente, solo los altos directivos están involucrados, pues tienen la imagen global de la empresa y de los contextos que influyen en la actividad (Chiavenato, 1993). Por su parte, el control de gestión se refiere fundamentalmente a la difusión de la misión y de los objetivos de la organización por los distintos niveles jerárquicos, para alinear las actividades, las áreas funcionales y los colaboradores con la empresa en su conjunto (Anthony \& Govindarajan, 2007).

Por tanto, la simbiosis entre el pensamiento estratégico y las acciones llevadas a cabo en los distintos niveles de operación de la cadena de mando, garantiza una alta participación y la responsabilización de todos en la actuación de la empresa, una uniformidad real de respuesta al mercado y a sus distintos participantes y el logro de los objetivos organizacionales.

Para cumplir su misión, el control de gestión se basa en tres tipos de instrumentos, que representan los diferentes aspectos de su metodología (Jordan, Neves \& Rodrigues, 2008):

- De pilotaje

- De orientación del comportamiento

- De diálogo

\section{A. Instrumentos de pilotaje}

Son los instrumentos de control de gestión que permiten divulgar la estrategia por los distintos niveles jerárquicos y áreas funcionales de la organización. Por medio de ellos, se asientan los objetivos de cada sector, departamento, unidad de negocio o división; se definen los planes de actividades y recursos necesarios; se cuantifican y se programan en el tiempo los objetivos y planes de acción; se evalúan los desempeños mediante la comparación de las predicciones con los resultados y midiendo el logro de los objetivos definidos.

Los productos que forman estos instrumentos son:

- Planos operativos

- Presupuestos

- Panel de bordo y cuadro de mando integral

- Control presupuestario

El plan operativo se realiza normalmente con un horizonte temporal de tres años y tiene como objetivo identificar las actividades y los recursos necesarios para alcanzar los objetivos de cada área y nivel jerárquico dentro de la organización (Jordan, Neves \& Rodrigues, 2008). Es fundamental que el gestor de cada sector participe en la preparación del plan operativo, porque nadie sabrá mejor que él el impacto de las decisiones sobre las actividades de su área particular. Además, su contribución garantizará que se identifique con los objetivos a lograr y que sea responsable ante la organización.

El principal objetivo del presupuesto es cuantificar y enmarcar temporalmente los objetivos definidos para cada área de la empresa. En conjunción con el plan operativo, el presu- 
puesto forma parte de los instrumentos de previsión. Por lo general, las previsiones se hacen para períodos anuales y tienen en cuenta las decisiones ya adoptadas en los planes de acción. El presupuesto representará la valoración financiera de los compromisos asumidos por el gestor y especificados en el plan operativo.

También es necesario llevar a cabo un seguimiento adecuado de los resultados y esta es la forma para determinar en qué medida los objetivos fijados se están logrando. Esa es la función del control presupuestario y del tableau de bord o cuadro de mando integral.

El control presupuestario se caracteriza por el seguimiento de la evolución de los datos financieros y se apoya en la contabilidad general y de costos. Por eso, es un instrumento de análisis pesado y de disponibilidad más lenta. Sin embargo, permite tener una idea perfecta del alcance de las previsiones financieras definidas y de la extensión de los desvíos existentes.

El panel de bordo y el cuadro de mando integral surgieron como complementos del instrumento anterior. De hecho, pretenden ser flexibles, disponibles y de rápida acción, pues su base son indicadores financieros y no financieros. Así, sus objetivos son dotar a los gestores de información personalizada de acuerdo a su área de trabajo, con datos de todo orden, pues los objetivos de la organización no son solo financieros. Además, es esencial que permitan que los gestores operativos actúen rápidamente sobre los desvíos existentes y es fundamental que estén fácilmente disponibles (Kaplan \& Norton, 2004).

En resumen, los instrumentos de pilotaje permiten a los gestores operativos reflexionar sobre los objetivos de su área de trabajo, para contemplar las actividades y recursos necesarios, y supervisar el progreso de los logros ante las predicciones realizadas. Fomentar su participación en el establecimiento de objetivos permite involucrar a toda la empresa en la estrategia desarrollada por la alta dirección y que la misión y los valores organizacionales se propaguen por ella.

\section{B. Instrumentos de orientación del comportamiento}

Un problema importante que surge en el control de gestión es asegurar que la organización funcione como un todo, o sea, que los objetivos y actividades de cada sector contribuyan al éxito global de la empresa. De hecho, con los instrumentos de pilotaje se promueve la participación de los gestores de operaciones en la estrategia a implementar. Sin embargo, debe asegurarse de que las acciones y decisiones de los gestores descentralizados cumplan los objetivos de la empresa y no solo los objetivos locales (Anthony \& Govindarajan, 2007). Este es el principal objetivo de los instrumentos de orientación del comportamiento. Para este propósito, se basan en tres productos:

- Centros de responsabilidad.

- Criterios para la evaluación del desempeño, utilizando medidas propias para los diferentes tipos de centros de responsabilidad.

- Sistemas de precios de transferencia interna.

Mediante la organización en centros de responsabilidad, se pretende definir con precisión el ámbito de actividad de cada gestor, al establecer objetivos, actividades y medios de que 
dispone y sobre los cuales tiene poder de decisión. La tipología del centro de responsabilidad varía según el grado de autonomía del gestor y puede tener, entre otras, las siguientes clasificaciones:

- Centros de costes: los gestores solo controlan costos de las actividades.

- Centros de resultados: los gestores controlan los ingresos y costos de la actividad.

- Centros de inversión: los gerentes, además de los resultados, controlan los activos y pasivos asociados a la actividad.

Los criterios para la evaluación del desempeño se aplican con base en la clasificación de los centros de responsabilidad, y es fundamental que el gestor se responsabilice solo por los medios sobre los cuales tiene poder de decisión. De lo contrario, el gestor sería evaluado sobre elementos que no están directamente relacionados con sus decisiones. Esta situación da lugar a comportamientos de rebeldía y de desmotivación de los responsables operativos, pues no se identifican con el modelo de control de gestión implementado, que termina perdiendo su utilidad.Así, los centros de costo deben ser evaluados teniendo en cuenta los costes reales antes que los estimados, y se deben tener como referencia los costes estándar que se definieron inicialmente, a fin de determinar los niveles de eficiencia alcanzados en la realidad. Los centros de resultados deben ser analizados por el margen de contribución que corresponde a la diferencia entre los ingresos y los costes directos del bien o servicio producido. Este sistema incorpora todos los ingresos y costos decididos por el centro de responsabilidad, lo que evita la repartición de los costos y la rebeldía de los gestores, ante una evaluación con indicadores de los cuales no son responsables. Por último, además de la evaluación de los ingresos y costos generados en los centros de inversión, es esencial analizar el impacto de las decisiones del gestor en el nivel de los activos y pasivos utilizados. Así, surge el concepto de activo económico que contiene el valor de los activos menos los pasivos derivados o asignados al centro (Neves, 2012). Así, por lo general, se utilizan indicadores que tratan de medir la capacidad de la empresa para crear valor ante el coste de capital necesario para financiar los activos económicos de cada centro. Entre estos indicadores, se destacan el EVA (economic value added) y el margen de contribución residual que se diferencia del anterior, al no considerar el efecto fiscal en los resultados de los centros de inversión (Neves, 2011).

Por último, los sistemas de precios de transferencia interna tienen por objetivo mejorar el intercambio de bienes y servicios realizados internamente entre los diferentes sectores de la empresa. Su implementación es crucial para orientar el desempeño de los gestores, ya que los lleva a optimizar los recursos de que disponen, a ser competitivos y a crear valor en la actividad que realizan. En este sentido, Charles T. Horngren, Srikant M. Datar y George Foster (2012) afirman que los métodos más utilizados por las empresas para determinar los precios de transferencia internos son los costos estándar, los costos estándar con margen y los precios de mercado, cuando hay referencias en el contexto empresarial. Sin embargo, los autores subrayan la importancia de que, independientemente de 
la metodología, el conjunto de precios de transferencia interna debe ser siempre el resultado de la negociación y de la aceptación por parte de los gestores involucrados.

Así, los instrumentos de orientación de comportamiento surgen para asegurar que la actividad de los gestores descentralizados contribuirá no solo a la consecución de los objetivos locales de cada área de trabajo, sino también a lograr los objetivos generales de la empresa. Básicamente, el uso de instrumentos de pilotaje deberá tener en cuenta el marco adecuado de los diferentes sectores en la clasificación de los centros de responsabilidad y sus criterios de evaluación de desempeño, y la definición más conveniente de los sistemas de precios de transferencias internas en cada caso particular.

\section{Instrumentos de diálogo}

El éxito del control de gestión y la eficacia de sus instrumentos son muy dependientes de la coordinación y de la participación de los diversos niveles jerárquicos y áreas de trabajo, en la diseminación por toda la organización de la misión y los objetivos establecidos por la alta dirección.

Por esto, es esencial que haya herramientas que permitan un alto flujo de comunicación y un diálogo frecuente e informal. Así, tradicionalmente, los instrumentos del diálogo se componen de salas de estar, fechas e informes de reuniones. Sin embargo, se destaca otro instrumento: la intranet. De hecho, la red electrónica interna de las organizaciones permite el intercambio rápido de información; hay muchos ejemplos de éxito en los principales grupos empresariales internacionales con la creación de verdaderas comunidades de intercambio de ideas y de resolución de problemas, lo que hace los procesos internos más eficientes y la capacidad de respuesta más rápida al cliente (Kluge, Stein \& Licht, 2002).

El control de gestión deberá actuar como un contrato entre la empresa y el gestor según su desempeño para el logro de los objetivos. Por eso, es fundamental que todos los interesados se sientan involucrados e identificados con las responsabilidades a asumir. Solo así podemos garantizar una uniformidad de acción y una verdadera unión en pro de la consecución de los objetivos organizacionales.

\section{El caso estudiado}

\subsection{La empresa}

Abordaremos el estudio de caso sobre Tecnológica S.A., una compañía del sector de las tecnologías de la información. La empresa se dedica a la ejecución de proyectos integrados de desarrollo de software, y proporciona una mezcla de marcas propias y tecnologías de referencia en el mercado mundial. Geográficamente, actúa en Portugal y se comprometió en una estrategia de internacionalización fuerte para los mercados emergentes de idioma portugués, a saber: Angola, Mozambique y Brasil. Tecnológica S.A. tiene una facturación anual de unos 18 millones de euros en Portugal. Sin embargo, si tenemos en cuenta el grupo de sociedades, incluidas las filiales de Brasil y África, la facturación supera los 60 millones de euros. Así, según los datos sectoriales disponibles para 2012, el grupo es uno de los 20 mayores del mercado portugués 
de tecnología de la información y uno de los 10 mejores, teniendo en cuenta solo las empresas de servicios en esta área de negocio.

\subsection{Las fases de desarrollo de la empresa}

Tecnológica S.A. fue fundada en 1990 y apareció como una empresa de software. Su proyecto inicial fue el desarrollo de un sistema de gestión integrado (conocido como ERP, enterprise resource planning). En esta etapa de su vida, la organización interna se basaba en una estructura simple e informal, con un órgano de dirección y un equipo flexible, que desempeñaba un poco de todas las funciones de la organización. Sin embargo, debido a la positiva evolución de los negocios, la estructura organizativa de forma rápida pasó a una estructura funcional con una mayor especialización de las tareas de los diferentes colaboradores.

En 1992, Tecnológica S.A. había desarrollado un conjunto de asociaciones tecnológicas y acuerdos estratégicos de distribución para la adquisición de conocimientos especializados en tecnología de la información, y había dejado de ser una empresa monoproducto. Este esfuerzo de diversificación fue acompañado por un cambio en la organización, que pasó a tener una estructura de divisiones por producto. En la práctica, el área comercial se dividió en varios gestores de producto que tenían objetivos comerciales claros y definidos. Esta opción se dirigió a un mercado más grande, ya que en este sector, el ciclo de vida de los productos es muy reducido, las necesidades de los clientes cambian rápidamente y hay una entrada constante de nuevos competidores.
Desde 1996, Tecnológica S.A. optó por enfatizar la oferta de servicios, por un lado, con el objetivo de reducir el grado de exposición y la dependencia de sus asociados tecnológicos y por el otro, para mejorar su valor añadido por empleado. En esta etapa de su vida, y como resultado de la innovación, del crecimiento y de la competitividad del sector de las tecnologías de la información, la empresa implementó una forma más avanzada de la estructura divisional, al constituir unidades estratégicas de negocio, UEN y unidades de soporte de negocio, USN.

El actual modelo de organización es una de las características clave de la empresa, ya que la existencia de las unidades estratégicas de negocio proporciona altos niveles de creatividad y motivación, derivadas de una mayor autonomía y descentralización de la autoridad. Este tipo de estructura se produjo como una forma de combatir las desventajas de la organización funcional, la que se traduce en relaciones burocráticas entre los diferentes departamentos, mayor número de niveles jerárquicos que retrasan la toma de decisiones, mayor formalización de los procedimientos y centralización del poder, lo que hace que la organización se torne inflexible ante el cambio y, finalmente, preste poca atención al mercado y a los consumidores, ya que hay una orientación clara para el funcionamiento interno y para el cumplimiento de las normas y de la cadena de mando.

Las unidades de negocio incluyen todas las funciones relacionadas con los productos o mercados, con la intención de obtener una respuesta más rápida a los consumidores y al mercado en general. Ellas son responsables de la planeación y la coordinación de todas las tareas 
relacionadas con los segmentos que atienden, desde la investigación hasta la asistencia del servicio posventa. Por eso, hay un enfoque constante en el medio ambiente y en los consumidores, lo que asegura una mayor vigilancia y control sobre los cambios externos. Esta característica de la estructura organizativa de Tecnológica S.A. es muy importante porque, en una industria con gran diversidad de ofertas de productos y servicios, permite a la compañía diversificar más fácilmente su negocio sin perder los índices de calidad en el servicio prestado. De hecho, dentro de la estructura organizacional, se crean verdaderas miniempresas especializadas. Así, cada unidad de negocio se centra en un núcleo de competencias tecnológicas y soluciones de negocio, que garantiza a sus clientes la competitividad y la calidad de sus servicios, sea con las características únicas de su oferta, sea con plazos reducidos de respuesta.

Las unidades de soporte de negocio tienen gestores propios y realizan actividades específicas como el marketing global de la empresa y los servicios de contabilidad y financieros.

Sin embargo, el resultado de la competitividad entre las unidades y del esfuerzo continuo para la obtención de los objetivos, ha desarrollado en los últimos años el espíritu de granja ${ }^{1}$, por lo que a veces es difícil asegurar la alineación de la acción entre las diferentes áreas de trabajo y los empleados. Por eso, la compañía ha invertido grandes cantidades de recursos para formalizar más los procesos y aplicar un sistema de evaluación y gestión de los proyec-

1 El espíritu de granja se presenta cuando los gestores se centran solo en los objetivos de sus unidades sin tener en cuenta el desempeño de la compañía. tos, que incluí en los criterios para el análisis de los empleados y de las unidades, la capacidad de trabajar en equipo y compartir conocimientos mediante su contribución a la realización de los proyectos.

\subsection{La planeación y el control en Tecnológica S.A.}

Con los años, se ha desarrollado la metodología para monitorizar la actividad, adaptar la información a la estructura de la organización y permitir una real delegación de autoridad. Así, en la actualidad, el presupuesto anual se desarrolla en una reunión denominada Kick-off, que se celebra durante dos días en noviembre en un hotel, y cuyo objetivo principal es el debate y la elaboración del presupuesto para el año siguiente.

En esa reunión, la administración presenta el plan estratégico de la empresa y todas las unidades discuten sus objetivos, planes de acción y también su presupuesto, precios de transferencia interna y los premios asociados a la consecución de los objetivos.

En cuanto a la preparación del presupuesto, hay algunos detalles relevantes:

- Cada unidad elabora su presupuesto.

- Se presentan los ingresos internos y externos, así como las distintas políticas comerciales que se espera poner en práctica (plazo medio de cobro y de pago y la política de existencias).

- Se describen los costos operativos que se prevén para el año, divididos en cinco categorías:

- Variables: están directamente relacionados con el volumen de negocios y el 
nivel de actividad. Hay coeficientes técnicos normalmente definidos.

- Directamente relacionados con los recursos utilizados en el negocio: la empresa utiliza los recursos humanos y materiales que producen y generan costes. Así, el presupuesto identifica de forma clara los costos relacionados con los empleados y con los activos asignados a cada unidad.

- En relación directa con los inversiones en marketing.

- Provenientes de transferencias internas que derivan de la utilización de los servicios producidos en otras unidades.

- Generales, como la electricidad, el agua, la higiene y la seguridad, que se distribuyen en función de criterios de asignación como el número de personas y el espacio físico ocupado por cada unidad.

- Su formato presenta una base mensual; después se consolidan los valores para todo el año.

- Representa un contrato entre el gestor y la empresa en una relación de compromiso con los objetivos presentados, para contribuir al éxito global de la empresa y en la definición de los premios que cada gestor recibirá al final de cada año.

Así, el sistema contable y financiero permite el diseño y control del presupuesto de cada unidad, lo que garantiza la preparación de sus estados financieros mensuales:

Así, desde el punto de vista económico, es posible controlar el resultado de cada uni- dad, al analizar el margen obtenido por cada gestor (margen de contribución de la unidad) y los ingresos netos generados después de la asignación de los costos de marketing y de la actividad general. En cuanto a la situación financiera, se verifica el nivel de inversión asociado a cada unidad, es decir, las fuentes de financiamiento utilizadas (autofinanciamiento y el capital de la deuda derivada de las condiciones de pago del ciclo de funcionamiento).

En ambas, el gestor puede ir siguiendo la evolución de la actividad en términos de valores acumulados y compararlos con las estimaciones iniciales de los resultados financieros. Aunque la desagregación de la información permita evaluar la situación económica y financiera de cada unidad, no permite destacar varios aspectos importantes en la evaluación de los gestores: la contribución de las unidades para los distintos proyectos en los que participan diferentes equipos, y el análisis del desempeño en variables no financieras vitales para el éxito sostenido de la empresa.

Así, como se mencionó anteriormente, se implementará un sistema de información que va a demostrar el desempeño de las unidades en los proyectos mediante el costeo basado en actividades y que va a permitir supervisar el logro de objetivos no financieros de acuerdo con la lógica del cuadro de mando integral, al establecer relaciones de causa y efecto entre los activos intangibles, los procesos internos, la satisfacción del cliente y el desempeño financiero. 
LA CONTRIBUCIÓN DE LOS SISTEMAS DE CONTROL DE GESTIÓN PARA EL ÉXITO EMPRESARIAL / N. DELICADO / 865

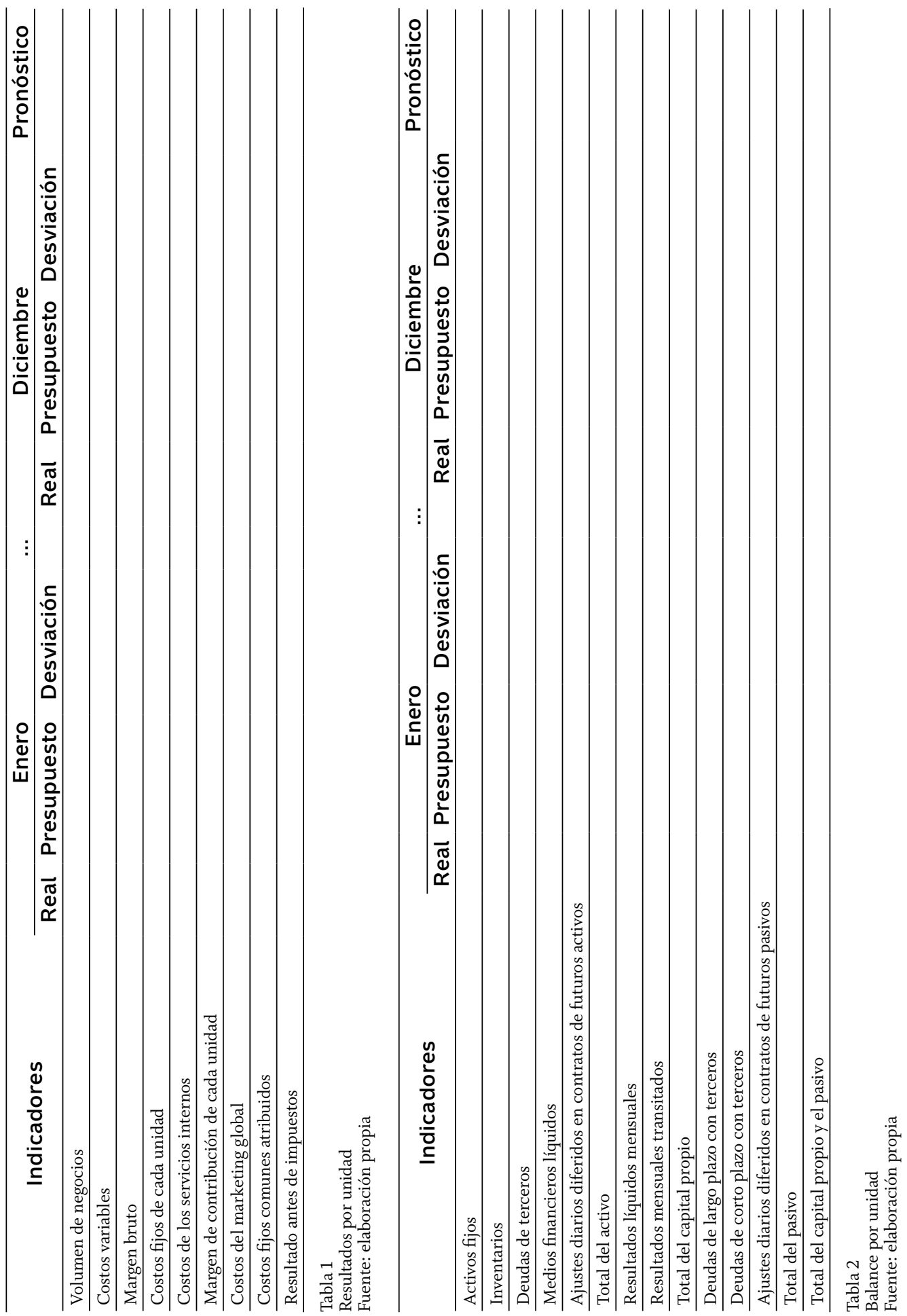




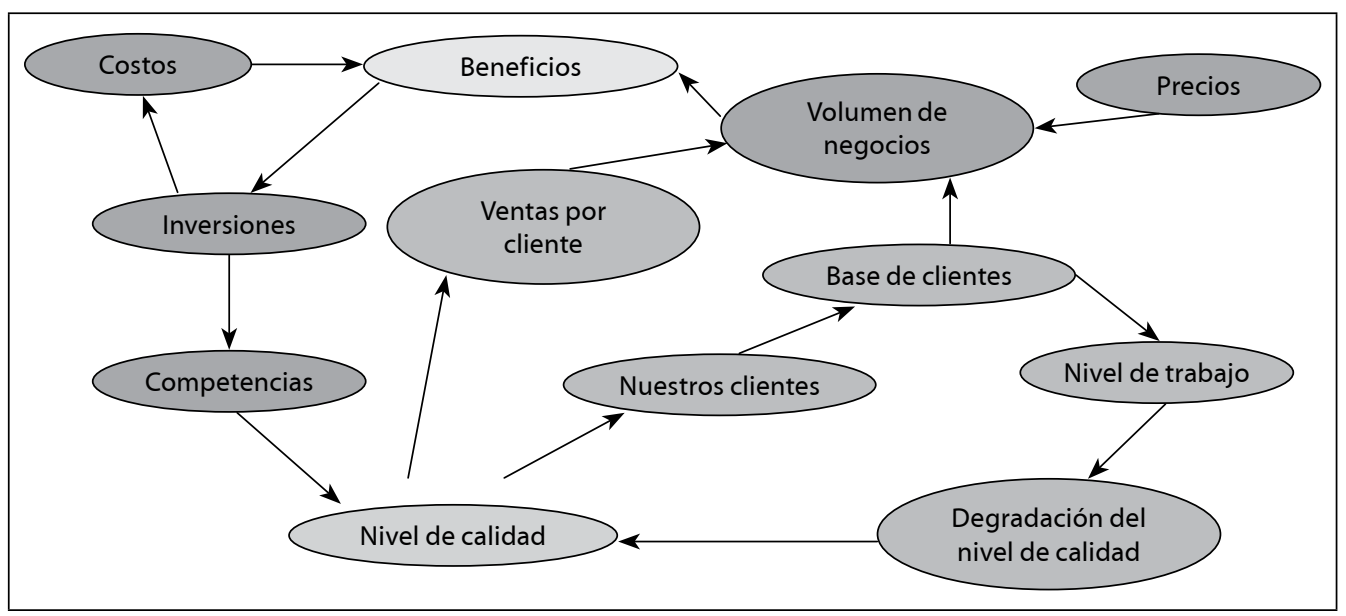

Gráfica 1

Ejemplo de un sistema dinámico

Fuente: adaptado de Amarildo da Cruz Fernandes (2003)

Se busca, por tanto, conocer cada vez mejor la actividad, los respectivos mercados que atienden y los proyectos que se ejecutan, y también romper con el espíritu de granja mediante la evaluación de la capacidad de los gestores para coordinar el funcionamiento de sus unidades con los objetivos generales de la empresa.

\subsection{Centros de responsabilidad de} Tecnológica S.A.

\subsubsection{Caracterización de unidades}

La empresa se compone de doce (12) unidades estratégicas de negocio y seis (6) unidades de soporte con las siguientes funciones principales:

- Unidades Estratégicas de Negocio, UEN: son responsables del área comercial y de producción de la empresa y coordinan las actividades necesarias para satisfacer el mercado.
- Unidad de soporte al negocio - Gestión del conocimiento: tiene la responsabilidad de asegurar la captura y el intercambio de conocimientos dentro de la organización, para dar una oportunidad permanente a la innovación en procesos, productos y servicios ofrecidos.

- Unidad de soporte al negocio - Administración y finanzas: es la responsable por todos los servicios de contabilidad, administrativos, legales y financieros de la empresa.

- Unidad de soporte al negocio - Marketing: es la responsable de la implementación del plan de marketing global de la empresa y de las actividades de promoción de las unidades.

- Unidad de soporte al negocio - Recursos estratégicos: es la responsable de la prospección de nuevas tecnologías y asociaciones, esenciales para el crecimiento sostenido.

- Unidad de soporte al negocio - Calidad: asegura el reclutamiento y selección de re- 
cursos humanos, y todas las actividades relacionadas con el control de calidad.

- Unidad de soporte de negocio - Infraestructura tecnológica: es la responsable por la gestión de las plataformas de trabajo de la empresa, y realiza el mantenimiento de los sistemas internos y externos, cuando las unidades de negocio lo soliciten.

Además de las unidades mencionadas, en la estructura organizativa están la administración y para el sistema de control de gestión, la unidad de eficiencia financiera. Sus principales funciones son:

- Administración: coordina las actividades de las unidades, para que los intereses locales converjan con los de la organización y garanticen la entrada de la empresa en los mercados objetivos.
- Eficiencia financiera: no tiene realidad física y sirve como el banco de las distintas unidades que constituyen la estructura de la organización. Suministra el capital necesario y los activos fijos.

\subsubsection{Tipos de centros de responsabilidad}

La definición de los centros de responsabilidad contribuye al desarrollo del espíritu emprendedor de los gestores y es un factor clave para el éxito de la organización. La importancia de estos pasa por dos puntos: su contribución, por una parte, a la eficiencia de la organización y, por otra parte, a evaluar igualitariamente el desempeño de los gestores. Por eso, teniendo en cuenta el poder de decisión de los gestores, se han definido los siguientes tipos de centros de responsabilidad:

\begin{tabular}{|c|c|c|c|}
\hline División & Control & Descripción & $\begin{array}{c}\text { Centro de } \\
\text { responsabilidad }\end{array}$ \\
\hline $\begin{array}{l}\text { Unidades } \\
\text { estratégicas de } \\
\text { negocio, UEN }\end{array}$ & $\begin{array}{l}\text { Necesidades de } \\
\text { capital circulante, } \\
\text { NFM } \\
\text { Rendimientos } \\
\text { Costos }\end{array}$ & $\begin{array}{l}\text { Los gestores negocian plazos de entrega y de pago y } \\
\text { definen la política de inventarios. } \\
\text { Los rendimientos dependen de las ventas al exterior y de } \\
\text { las transacciones internas. } \\
\text { Los costos dependen de su actividad normal y de las } \\
\text { transacciones internas. }\end{array}$ & Centros de inversiones \\
\hline $\begin{array}{l}\text { Unidades de } \\
\text { soporte de } \\
\text { negocio, USN }\end{array}$ & Rendimientos & $\begin{array}{l}\text { Los rendimientos dependen de las transacciones } \\
\text { internas. } \\
\text { Los costos dependen de su actividad normal y de las } \\
\text { transacciones internas. }\end{array}$ & Centros de resultados \\
\hline Administración & Rendimientos & $\begin{array}{l}\text { Los rendimientos dependen de las transacciones } \\
\text { internas. } \\
\text { Los costos dependen de su actividad normal y de las } \\
\text { transacciones internas }\end{array}$ & Centro de resultados \\
\hline $\begin{array}{l}\text { Eficiencia } \\
\text { financiera }\end{array}$ & Rendimientos & $\begin{array}{l}\text { Examina todos los activos fijos propiedad de la empresa y } \\
\text { alquilados por una renta económica. } \\
\text { Los rendimientos dependen de las transacciones internas } \\
\text { y de las aplicaciones financieras de la empresa. } \\
\text { Los costos dependen de su actividad normal y de las } \\
\text { transacciones internas. }\end{array}$ & Centro de resultados \\
\hline
\end{tabular}

Tabla 3

Tipos de centros de responsabilidad en Tecnológica S.A.

Fuente: elaboración propia 
En la clasificación asignada, se destacan los siguientes hechos:

- Hemos considerado las unidades de negocio como centros de inversión, porque los gestores tienen el poder de tomar decisiones en materia de política comercial y de inventarios, y deben ser evaluados teniendo en cuenta las necesidades o los recursos financieros creados.

- Consideramos las unidades de soporte y la administración como centros de resultados, ya que solo tienen poder de decisión sobre rendimientos y costos de su actividad. $\mathrm{Al}$ igual que las unidades estratégicas de negocio, UEN, las unidades de soporte y la ad- ministración no tienen ninguna influencia en la forma en que se adquiere el activo fijo, luego arrendado, lo que es decisión de la unidad de eficiencia financiera.

- Se consideró la unidad de eficiencia financiera como centro de resultados. Debido a que administra activos, también se podría considerar como un centro de inversión. Sin embargo, el costo de los activos fijos en su poder está soportado por la unidad de eficiencia financiera; su valorización surge de los costos de sus actividades y puede asumir la forma de costos de oportunidad o interés. Consideremos el siguiente ejemplo:

\begin{tabular}{llll}
\hline \multicolumn{2}{c}{ Balance de la eficiencia financiera } \\
\hline \multicolumn{2}{c}{ Inversiones } & \multicolumn{2}{c}{ Fuentes de financiamiento } \\
\hline Activos fijos & 50 & Resultado del ejercicio & 62,56 \\
\hline Aplicaciones & 33,6 & Capital & 20 \\
\hline Medios financieros líquidos & 18,96 & Préstamo bancario & 20 \\
\hline Total de inversiones & 102,56 & Total de financiamientos & 102,56 \\
\hline
\end{tabular}

\begin{tabular}{lclc}
\hline \multicolumn{4}{c}{ Cálculo del margen del centro de eficiencia financiera } \\
\hline \multicolumn{2}{c}{ Opción: tratar como centro de resultados } & Opción: tratar como centro de inversión \\
\hline Resultado operacional & 62,56 & Resultado operacional & 62,56 \\
\hline Costo financiero del préstamo, 8\% & 1,60 & Activo & 102,56 \\
\hline Costo de oportunidad del capital, 10\% & 2,00 & Costo del activo - 3,51\% (WACC) & 3,60 \\
\hline Margen de contribución & 58,96 & Margen de contribución residual & 58,96 \\
\hline
\end{tabular}

Tabla 4

Determinación de la creación de valor de la eficiencia financiera

Fuente: elaboración propia

Para determinar la contribución de cada centro de responsabilidades con sus propias fuentes de financiación, es indiferente la clasificación como centros de resultados o de inversión.
Sin embargo, en la actualidad, las unidades de negocio son tratadas de un modo más cercano a la clasificación de los centros de resultados. Si bien hay una preocupación con la 
creación de valor y el retorno de las inversiones, la evaluación de los centros se basa en la lógica de los costos y los ingresos, representada por los dos ejemplos que presentamos a continuación:

- Los activos fijos no son patrimonio de las unidades de negocio. Estas le pagan el coste de su uso a la unidad de eficiencia financiera con una renta económica, que simula el financiamiento de la adquisición de los bienes. Así, tenemos la intención de verificar si las unidades tienen la capacidad de autofinanciarse para pagar las inversiones realizadas.

- Siempre que las unidades de negocio tengan saldos de caja negativos, pagan a la empresa intereses por las necesidades financieras creadas.

Como se mencionó anteriormente, para garantizar el acceso de los gestores a estos datos se presentan los resultados financieros mensuales de cada unidad: cuenta de resultados, balance general y estado de flujo de efectivo.

\subsection{Evaluación de los centros de responsabilidad}

El actual modelo de evaluación de Tecnológica

S.A. presenta las siguientes características:

- Solo las unidades de negocio son evaluadas.

- La evaluación de las unidades de negocio se realiza con los siguientes cálculos:

\section{Resultado operacional de la unidad}

\begin{tabular}{l}
\hline - Costos generales atribuidos \\
\hline - Prestaciones internas de las unidades restantes \\
\hline - Renta económica de los activos utilizados \\
\hline - Costo del capital calculado sobre los saldos de medios financieros negativos \\
\hline Resultado de la unidad \\
\hline
\end{tabular}

De este modo, tenemos la intención de evaluar su desempeño en cuatro perspectivas:

- Capacidad de generar excedentes financieros frente a los costos de su actividad.

- Capacidad para cubrir los costos necesarios para el funcionamiento de las unidades de apoyo y de las instalaciones.

- Capacidad financiera para pagar las inversiones en el desarrollo de su actividad.

- Castigar la creación de necesidades financieras que sean consecuencia de mala gestión de tesorería.
Como ya se dijo, de acuerdo con la autoridad de los gestores de las diferentes unidades, podemos definir dos tipos de centros de responsabilidad en la empresa: de resultados y de inversión.

Los centros de resultados deben ser evaluados por el margen de contribución, incluidos los ingresos y los costes sobre los que el gestor tiene poder de decisión. En cuanto a los centros de inversión, como el gestor también podrá decidir sobre los activos y pasivos, la evaluación debe basarse en el margen de contribución residual (o EVA, si se quiere in- 
corporar el efecto fiscal), incluyendo solo los elementos en los que el gestor tiene responsabilidades. Siguiendo esta lógica, presentamos la lista de centros de responsabilidad y los indicadores de evaluación que debe aplicar Tecnológica S.A.:

\begin{tabular}{lll}
\hline \multicolumn{1}{c}{ Unidad } & \multicolumn{1}{c}{$\begin{array}{c}\text { Tipo de centro de } \\
\text { responsabilidad }\end{array}$} & Indicador de evaluación \\
\hline Unidades estratégicas de negocios, UEN & Centros de inversiones & Margen de contribución residual \\
\hline Gestión de conocimiento & Centro de resultados & Margen de contribución \\
\hline Administrativa y financiera & Centro de resultados & Margen de contribución \\
\hline Marketing & Centro de resultados & Margen de contribución \\
\hline Recursos estratégicos & Centro de resultados & Margen de contribución \\
\hline Cualidad & Centro de resultados & Margen de contribución \\
\hline Infraestructura tecnológica & Centro de resultados & Margen de contribución \\
\hline Administración & Centro de resultados & Margen de contribución \\
\hline
\end{tabular}

Tabla 5

Indicadores a utilizar en la evaluación de los centros de responsabilidad

Fuente: elaboración propia

En relación con el actual sistema de evaluación de los centros de responsabilidad, hacemos las siguientes observaciones:

- Los gastos generales de la empresa en las distintas unidades se asignan con claves de reparto. El gestor no tiene poder de decisión sobre ellos. Sin embargo, el resultado de la unidad que coordina se ve afectado directamente por el valor de estos indicadores.

Así se generan conflictos y sentimientos de desconfianza entre los gestores de las UEN y el responsable administrativo y financiero; los valores imputados se confieren a los detalles.

- Los métodos para medir el costo del capital utilizado por cada unidad son la cobranza de la renta económica por la utilización de bienes y el interés por la existencia de saldos negativos de medios financieros. Estos métodos son menos punitivos para los gestores que la adopción del margen de contribución residual. A continuación, se presenta un ejemplo numérico con el fin de verificar esta situación:

\section{Resultado operacional antes de amortizacio-} nes: $1.000 €$

Activo: $100 €$

Tasa de amortización: 12,5\%

Opción: margen de contribución residual

Resultado operacional: 1.000 - $(100 \times 0,125)$ $=987,50 €$

Tasa de costo de capital: $25 \%$

Margen de contribución residual: 987,50 $[(100-12,5) \times 0,25]=965,63 €$

Opción: renta económica

Vida útil del activo: 8 años

Tasa del costo de capital: $25 \%$ 
Valor de adquisición: $100 €$

Término de la renta pospagada para 8 años a una tasa de interés de $25 \%$ por período: $30,04 €$ Resultado operacional corregido: 1.000 - 30,04 $=969,96 €$

Así, verificamos que el margen de contribución residual penaliza más el resultado de la unidad que la renta económica. En cuanto a los intereses cobrados por los saldos negativos de los recursos financieros, es importante mencionar que si tenemos en cuenta los medios financieros en activos, el valor que se cobrará a las unidades es idéntico en ambas metodologías. Considere los siguientes ejemplos:

\begin{tabular}{lclc}
\hline \multicolumn{4}{c}{ Balance de la unidad de negocio XX } \\
\hline Inversiones & \multicolumn{2}{c}{ Fuentes de financiamiento } \\
\hline Clientes & 3.400 & Resultado del ejercicio & 2.000 \\
\hline Medios financieros & $(1.200)$ & Proveedores & 200 \\
\hline Total de inversiones & 2.200 & Total de fuentes de financiamiento & 2.200 \\
\hline
\end{tabular}

Tabla 6

Determinación del balance con medios financieros negativos Fuente: elaboración propia

En este caso, los medios financieros podrían considerarse como un préstamo de la propia empresa a la unidad. Como tal, pasaremos a tener los siguientes valores para el margen de contribución residual:

Costo del capital propio: $25 \% \rightarrow$ costo a asociar a la utilización de capital de la empresa por vía de los medios financieros negativos Capitales invertidos: $3.400-200=3.200 €$ Costo medio ponderado del capital $=$ $(2.000 / 3.200) * 0 \%+(1.200 / 3.200) \times 0,25=$ 0,094

Margen de contribución residual $=2.000-$ $(3.200 \times 0,094)=1.700 €$

El resultado de la unidad representa su capacidad de autofinanciamiento, pero no por eso debe tener cualquier costo de capital asociado. Al aplicar la metodología de la empresa, obtenemos el mismo resultado, en virtud de que el cálculo es idéntico:

Interés a imputar a la unidad: $1.200 * 0,25=300 €$ Resultado de la unidad: $2.000-300=1.700 €$

Vamos a ver ahora, un ejemplo con medios financieros positivos: 


\begin{tabular}{llll}
\hline & Balance de la unidad de negocio $\mathbf{X X}$ \\
\hline Inversiones & \multicolumn{2}{l}{ Fuentes de financiamiento } \\
\hline Clientes & 400 & Resultado del ejercicio & 2.000 \\
\hline Medios financieros & 1.800 & Proveedores & 200 \\
\hline Total de inversiones & 2.200 & Total de fuentes de financiamiento & 2.200 \\
\hline
\end{tabular}

Tabla 7

Determinación del balance con medios financieros positivos

Fuente: elaboración propia

Los valores para el cálculo del margen de contribución residual son los siguientes:

Costo del capital propio: $25 \%$

Capitales invertidos: $2.200-200=2.000 €$

Costo medio ponderado del capital $=$

$(2.000 / 2.000) \times 0 \%=0$

Margen de contribución residual $=2.000-0$

$=2.000 €$

En nuestra opinión, aunque no hacen parte del activo económico, los recursos financieros deben incluirse en el valor de los activos a ser financiados, ya que representan valores monetarios que se están utilizando sin la rentabilidad adecuada. Como tal, la metodología utilizada por la empresa, en referencia a los saldos de efectivo, cumple los objetivos de creación de valor.

Sin embargo, de acuerdo con los ejemplos presentados relativos a la renta económica, pensamos que sería conveniente aplicar los conceptos teóricos del margen de contribución y del margen de contribución residual, para determinar la capacidad de las distintas unidades para crear valor en sus negocios.

\subsection{Las transferencias internas de Tecnológica S.A.}

En cuanto a la actividad interna de la empresa, hay varios tipos de servicios que se pueden clasificar en los siguientes niveles:

Servicios de coordinación general y de acceso a bienes y capital

- Servicios de asesoramiento prestados por la administración a las unidades de negocio y unidades de apoyo.

- Servicios financieros y de alquiler de equipos por parte de la unidad de eficiencia financiera para la administración y las distintas unidades.

Servicios entre las unidades de negocio y de soporte

- Los servicios de apoyo proporcionados por las unidades de apoyo a las unidades de negocio.

- Los servicios prestados por las unidades de negocio para apoyar a las unidades de soporte, para mantener las instalaciones de trabajo. 


\section{Servicios entre las unidades de negocio y} unidades de soporte

En este nivel, es fundamental tener en cuenta que los servicios entre las unidades de negocios pueden tomar la forma de ventas al exterior y de apoyo a los proyectos en pre y posdesarrollo.

A continuación, se muestran de forma esquemática las relaciones internas en la Tecnológica S.A.

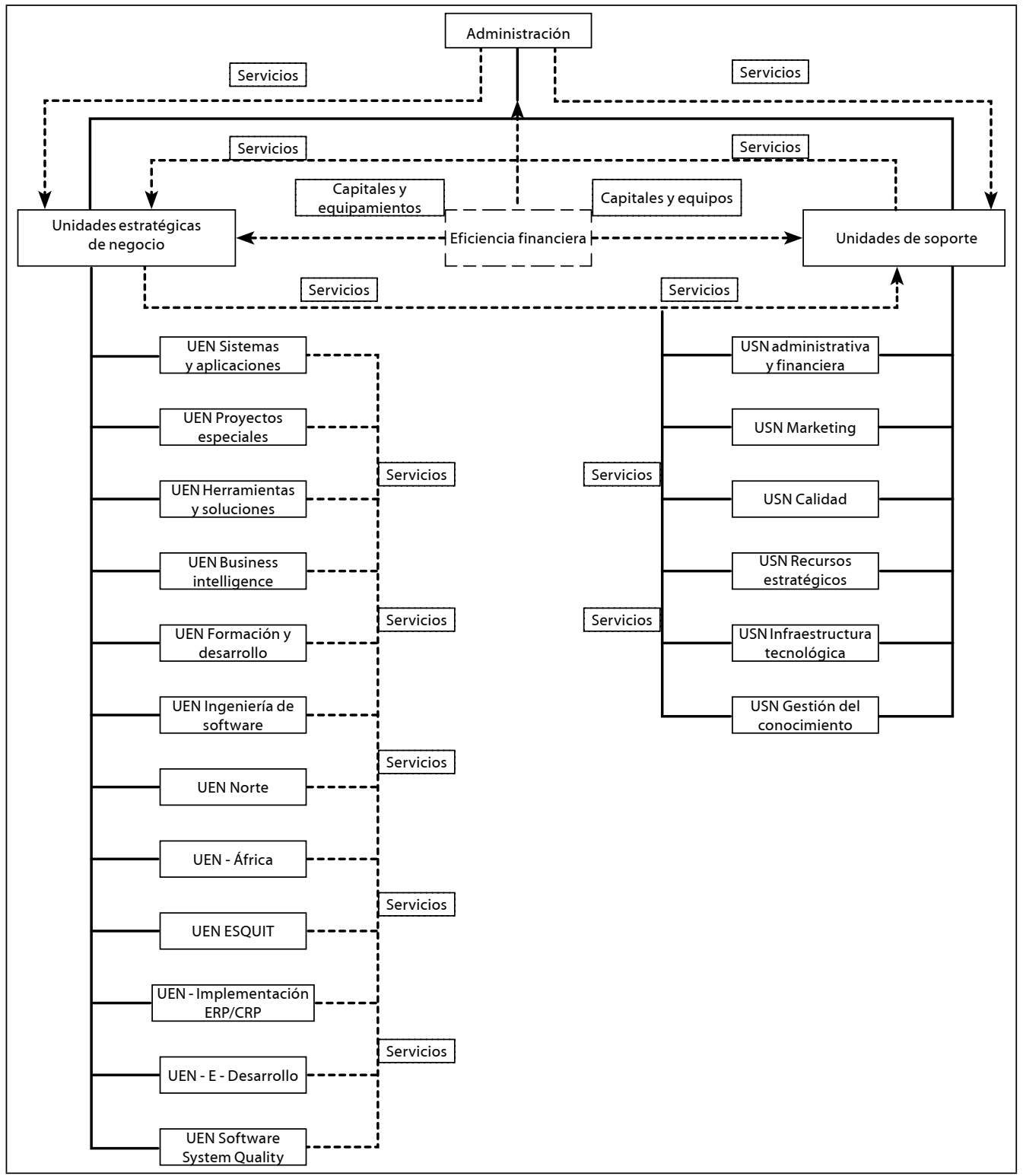

Gráfica 2

Prestaciones de servicios internos

Fuente: elaboración propia 


\subsection{Precio de las transferencias internas, PTI}

Actualmente, las transferencias internas se valorizan del siguiente modo:

\begin{tabular}{|c|c|c|}
\hline $\begin{array}{l}\text { Centro de } \\
\text { responsabilidad }\end{array}$ & Servicios realizados & Precio pactado \\
\hline UEN & $\begin{array}{l}\text { Servicios de manutención y desarrollo } \\
\text { interno }\end{array}$ & Precio de mercado \\
\hline $\begin{array}{l}\text { USN - Gestión del } \\
\text { conocimiento }\end{array}$ & $\begin{array}{l}\text { Servicios de formación y de desarrollo de } \\
\text { competencias }\end{array}$ & Precio negociado \\
\hline $\begin{array}{l}\text { USN - Administrativa y } \\
\text { financiera }\end{array}$ & $\begin{array}{l}\text { Servicios financieros, legales y de } \\
\text { contabilidad }\end{array}$ & $\begin{array}{l}\text { Tasa negociada sobre los costos fijos de cada } \\
\text { unidad }\end{array}$ \\
\hline USN - Marketing & $\begin{array}{l}\text { Servicios de implementación del } \\
\text { marketing institucional } \\
\text { Servicios de implementación de las } \\
\text { acciones promocionales de las UEN }\end{array}$ & $\begin{array}{l}\text { Tasa negociada sobre el volumen de negocios } \\
\text { de cada UEN } \\
\text { Costo real de las acciones }\end{array}$ \\
\hline USN - Recursos estratégicos & $\begin{array}{l}\text { Obtención de servicios de consultoría en } \\
\text { compañías y negocios }\end{array}$ & Precio negociado \\
\hline USN - Calidad & $\begin{array}{l}\text { Servicios de garantía de calidad } \\
\text { Servicios de reclutamiento y selección }\end{array}$ & Precios negociados \\
\hline $\begin{array}{l}\text { USN - Infraestructura } \\
\text { tecnológica }\end{array}$ & $\begin{array}{l}\text { Servicios de comunicaciones fijas } \\
\text { Servicios de alquiler de las instalaciones } \\
\text { tecnológicas } \\
\text { Servicios de fotocopias, impresiones y fax }\end{array}$ & $\begin{array}{l}\text { Costo real directo de cada UEN atribuido por } \\
\text { la extensión usada } \\
\text { Precio negociado } \\
\text { Costo real directo de cada UEN atribuido por } \\
\text { el código usado }\end{array}$ \\
\hline Administración & Servicios de consultoría a las unidades & $\begin{array}{l}\text { Precios negociados. Se atribuyen valores a los } \\
\text { gestores de las unidades de negocio - GUN } \\
\text { y a los colaboradores de las unidades de } \\
\text { negocio, mediante el porcentaje aplicado al } \\
\text { Valor Agregado Bruto (VAB) y al volumen de } \\
\text { negocios de cada una }\end{array}$ \\
\hline Eficiencia financiera & $\begin{array}{l}\text { Servicios de alquiler de equipos e } \\
\text { instalaciones } \\
\text { Servicios financieros - fuentes de } \\
\text { financiamiento }\end{array}$ & $\begin{array}{l}\text { Renta económica, teniendo en cuenta el valor } \\
\text { de adquisición, la vida útil y el costo de la tasa } \\
\text { de la inversión negociada } \\
\text { Renta negociada en alquiler de las } \\
\text { instalaciones, considerando el número de } \\
\text { personas de cada UEN } \\
\text { Tasa negociada del costo del capital utilizado } \\
\text { por las UEN }\end{array}$ \\
\hline
\end{tabular}

Tabla 8

Valorización de las transferencias internas

Fuente: elaboración propia 
De este modo, la empresa utiliza diversos tipos de precios de transferencia interna, a saber:

- Precios de mercado

- Precios negociados

- Costo real

Como sabemos, los precios de transferencia interna representan un vehículo para condicionar las actividades de los gestores operacionales, de acuerdo con los objetivos de la empresa (Horngren, Datar \& Foster, 2012). En Tecnológica S.A. se definen con este enfoque, pues se busca asegurar que las unidades contribuyen simultáneamente a los objetivos locales y globales. A continuación, se muestra la justificación de las distintas situaciones descritas en la tabla anterior:

- Los precios de mercado utilizados por la UEN en las transferencias internas buscan asegurar su competitividad, ya que las unidades de apoyo podrán contratar los mismos servicios en el exterior, en caso de que encuentren proveedores con mejores precios o condiciones comerciales.

- Los precios negociados en la valoración de los servicios prestados por unidades de soporte, tienen como objetivo cubrir los gastos de esas actividades y contribuyen a la rentabilidad de la empresa en primer lugar; y en segundo lugar, a garantizar que todos los gestores aceptan las reglas del juego y contribuyen al éxito de la estrategia de la empresa.

- La valoración de los servicios administrativos y financieros, que consiste en la aplicación de una tasa negociada en los costos fijos de cada unidad, quiere reducir el ries- go del negocio, lo que lleva a los gestores a optimizar de manera más eficaz su estructura de costos.

- La valoración de los servicios de marketing institucional se realiza con una tasa negociada sobre el volumen de negocios de cada unidad y parte del principio de que este está directamente relacionado con la reputación de la empresa.

- El costo real solo se aplica sobre los servicios que se pueden asignar directamente a los costos y que no hay peligro de que las unidades sean perjudicadas con las ineficiencias de los proveedores internos.

- La valoración de los servicios de consultoría de gestión dirigida a penalizar los gerentes que requieren compromiso por parte de los administradores, se centra en el número de personas, y en el valor creado por sus propias unidades.

- El alquiler de equipos por una renta económica simula el financiamiento de esos activos y se centra sobre las unidades que los utilizan; los intereses y las amortizaciones del capital son más rápidas que la depreciación fiscal, lo que comprueba que las unidades tienen capacidad de autofinanciamiento.

- El costo de capital definido busca penalizar las unidades generadoras de las necesidades financieras de la empresa pues normalmente la tasa negociada es bastante alta en comparación con las que prevalecen en el mercado financiero.

Teniendo en cuenta esta descripción, es posible identificar una serie de directrices que están 
detrás de la actual metodología utilizada para definir los precios de transferencia interna:

- Crear competitividad interna conduce a la existencia de un espíritu de innovación, lo que obliga los gestores a centrar sus actividades en las necesidades de los clientes, tanto internos como externos.

- Asegurar que las UEN presentan altas tasas de rentabilidad, capaces de cubrir los costos de capital derivados de los compromisos financieros provenientes de las inversiones constantes, característica del sector de tecnología de la información.

- Garantizar un riesgo de negocio reducido mediante una estructura de costos más racional, para evitar los cargos fijos, que podrán presionar la tesorería durante los períodos de menor actividad.

- Fomentar la delegación de la autoridad e involucrar a los gestores en los objetivos organizacionales, para que la administración se dedique a actividades estratégicas y los gestores operacionales se concentren en las oportunidades de mercado y la creación de valor para los clientes.

Sin embargo, en nuestra opinión, hay algunas situaciones que pueden ser optimizadas:

- Los precios de mercado deben ajustarse teniendo en cuenta las sinergias comerciales derivadas de los proveedores internos, para fomentar la realización de las transferencias internas a favor de la contratación de proveedores externos.

- Los servicios de las unidades de soporte también pueden ser valorados a precios de mercado, y los gestores se pueden orien- tar por la rentabilidad y no solo por la eficiencia de costes. Esto porque los gestores de las USN actualmente no muestran una preocupación empresarial en la manera en que gestionan sus centros de actividad, pues solo tienen cuidado con la cantidad de costos. Por eso, teniendo en cuenta los posibles riesgos de los PTI (encarecimiento de los servicios internos y una mayor demanda de proveedores externos), en las entrevistas realizadas fue posible identificar un cierto optimismo sobre el establecimiento de precios de mercado ajustados para las transferencias realizadas por las USN, lo que hace más objetivos los criterios y favorece el enfoque empresarial en la gestión de las USN, porque el desempeño de sus gestores sería más fácil de medir y de ser reconocido por la organización.

- Las UEN no deberían ser obligadas a adquirir los servicios a las unidades de soporte, ya que de esta manera se penaliza su rentabilidad si presentan costos más altos que los del mercado. Además, así se favorece indebidamente la actividad de las unidades de soporte que, sin ser competitivas, han garantizado clientes.

- No debería haber tasas de imputación de los costes de las unidades de soporte a las unidades estratégicas de negocio. Por ejemplo, el criterio del volumen de negocios utilizado para asignar el costo de marketing institucional es muy injusto, porque cuanto más facture la unidad, mayor es el costo que se aplicará, sin tener en cuenta la capacidad comercial del gestor. En estos casos, debe haber una forma de asignar el valor corres- 
pondiente a las UEN o, simplemente, se debe considerar como costos de la actividad general.

Estos cambios propuestos están dirigidos a dos objetivos principales:

- La creación de un espíritu empresarial en todos los ámbitos de actividad, para contribuir a la consecución de objetivos de la organización mediante una acción corporativa en todas las unidades, incluso en las de soporte, para fomentar la innovación y la calidad de los servicios y la rentabilidad de las inversiones. El mejor funcionamiento interno refuerza las competencias centrales de la empresa y garantiza el éxito del negocio.

- Responsabilizar a los gestores solo de los gastos sobre los que tienen poder de decisión, para involucrarlos en el sistema de control de gestión implementado y combatir cualquier desconfianza e indignación con los criterios utilizados en la asignación de los costos de las unidades de soporte. A propósito, este fue uno de los problemas que el director financiero destacó en el sistema de control de la gestión actual. En las reuniones mensuales entre él y los gestores de las unidades de negocio, es frecuente cuestionar la validez de las diversas asignaciones de gastos realizados (por ejemplo, los costos de las unidades de apoyo administrativo y financiero y de marketing), y poner en duda los valores de los estados financieros mensuales, ya que sus costes surgen sobrevalorados, lo que reduce los márgenes de sus centros.
La implementación de los precios de mercado ajustados se puede facilitar con la aplicación simultánea de la metodología de costeo basado en actividades, ya que hace visible el costo unitario de los servicios prestados por unidades de soporte, por lo que es más fácil negociar entre los distintos centros de responsabilidad, lo que crea una mayor motivación de los gestores sobre el sistema de control de gestión implantado.

\subsection{El diálogo como un factor crítico de éxito de Tecnológica S.A.}

Hay un especial cuidado en la empresa con la divulgación de la estrategia y con la participación de todo su personal en el logro de los objetivos organizacionales.

Como se dijo anteriormente, la planeación se formaliza en la reunión anual Kick-off, donde todos están presentes, desde los administradores hasta los empleados de nivel jerárquico más bajo. En esta reunión, que por lo general se lleva a cabo durante tres días, la administración presenta la visión y el plan estratégico de la empresa y los gestores de las unidades presentan los diferentes planes de negocios y presupuestos. Así, todos pasan a conocer el camino que la empresa recorrerá en los próximos años y qué papel desempeñarán en el éxito organizacional. Con el fin de crear ejemplos de "campeones", todos tienen una camisa de la empresa cuyo color varía según la antigüedad del empleado en la organización. Así, los colaboradores más recientes tienen la ambición de ganar el derecho a las camisas que representan una mayor antigüedad.

Además, el control presupuestario se realiza en reuniones periódicas que han definido 
reglas conocidas por todos. El éxito del sistema de control de gestión depende de la participación de todos en la definición y negociación de los objetivos a alcanzar, y de la interconexión de las distintas áreas funcionales, de modo que la información y los procesos fluyan de manera natural dentro de la organización. Por eso no es de extrañar el especial cuidado con este tema. Así, hay reglas establecidas, ya sea para la preparación de reuniones, ya sea por la propia forma en que ocurren. A continuación, se muestran las reglas actualmente vigentes:

\section{Preparación de la información disponible}

- La unidad administrativa y financiera prepara los estados financieros de cada unidad: cuenta de resultados, flujo de efectivo y balance.

- Una semana antes de la reunión, se actualizan todos los resultados del mes anterior, para que cualquier gestor pueda consultarlos.

- Antes de la reunión mensual, cada gerente se reúne durante una hora con el responsable de la unidad administrativa y financiera para analizar y validar la situación mensual.

\section{Reuniones}

- La frecuencia de las reuniones es mensual, con una duración de 10 horas: de las 8 a.m. hasta las 6 p.m.

- No se aceptan retrasos. Cualquiera que llegue después de la hora prevista, paga una multa.

- Están presentes la administración y todos los gestores de las distintas unidades.
- Se analizan los resultados globales de la empresa y de todas las unidades, en comparación con el mes anterior.

- Todos los gestores presentan sus planes de negocio actualizados.

- Se celebra el foro GUN (gestores de las unidades de negocio), un espacio para el debate y el intercambio de información entre los responsables de las unidades de negocio.

- Participan los invitados y después la administración termina la reunión.

Por último, además de la planeación y del seguimiento periódico, con mucha intervención de la administración y de los gestores, la empresa ha realizado esfuerzos para hacer un mayor intercambio de conocimientos. Hay tres herramientas muy utilizadas en este sentido:

- Newsletter de la empresa: los gestores y empleados de las empresas tienen el reto de escribir artículos mensuales sobre las experiencias en la organización.

- Repositorio de proyectos y foros de discusión: se puede encontrar información diferente acerca de los problemas y desafíos que se enfrentan en los proyectos, para evitar que las distintas unidades trabajen en proyectos similares y no compartan información.

- Formación: a cargo de gestores o colaboradores técnicos de las unidades sobre los proyectos que se han desarrollado con gran éxito.

En nuestra opinión, estos instrumentos de diálogo son la clave para el éxito del sistema de control de gestión, ya que no solo permiten la evaluación del desempeño con mayor claridad, sino el intercambio de conocimientos entre los 
diferentes responsables, lo cual crea sinergias en respuesta a los problemas de la vida cotidiana de la empresa.

\section{Conclusiones}

Con este trabajo, se ratificó que los sistemas de control de gestión pueden ser una herramienta poderosa para el éxito del negocio mediante la divulgación de la estrategia en la organización, pero también al involucrar a los colaboradores con los objetivos globales.

En primer lugar, el papel del presupuesto como una herramienta clave en la planeación y control de la actividad, depende de la capacidad de la empresa para adaptar su estructura organizativa a las necesidades de los sistemas de información. Durante su crecimiento, Tecnológica S.A. cambió su organización interna y su sistema de información; en la actualidad, la información por segmentos permite la obtención de los datos económicos y financieros de cada unidad. Fruto de la necesidad de una mayor cohesión organizativa, la empresa está implementando la gestión de proyectos y el cuadro de mando integral, lo que favorece la evaluación del desempeño con base en los indicadores de las unidades y en función de los objetivos organizacionales. Espera así eliminar el espíritu de granja y lograr la alineación de acción entre las distintas unidades de negocio y soporte, para que el presupuesto sea controlado en tres niveles: empresa, unidades y proyectos.

También se evidenció la importancia de la definición de los centros de responsabilidad con el fin de facilitar la toma de decisiones y la respuesta de las empresas en el contexto actual. Así, se mostró también que los indicadores deben ser seleccionados para la evaluación de los centros de responsabilidad de acuerdo con el medio sobre el que el gestor tiene poder de decisión. Con el desarrollo del estudio de caso, hemos sido capaces de obtener las siguientes conclusiones:

- Los centros de responsabilidad (unidades de negocio y de soporte), que actualmente representan una de las competencias básicas de la empresa, permiten una mayor velocidad y flexibilidad para responder al mercado, gracias a un mayor conocimiento del mercado por parte de los gestores y sus equipos. Por otra parte, la descentralización del poder de toma de decisiones también permitió la diversificación de negocio, ya que las unidades de negocio están desarrollando habilidades a medida que identifican oportunidades en el mercado.

- También se ratificó que los criterios para evaluar el desempeño de los centros de responsabilidad se basan en la creación de valor; hay una preocupación con la capacidad de las distintas unidades de negocio para cubrir los costos de sus actividades, de los servicios internos prestados por unidades de soporte y de los saldos de caja negativos.

- Por último, la evaluación de los resultados financieros en todos los ámbitos de la empresa es fundamental para crear una lógica empresarial en todos los procesos de trabajo y asegurar la concentración, tanto en los clientes internos como externos.

En este sentido, los precios internos de transferencia pueden tener un papel impor- 
tante al orientar el comportamiento de los gestores de actividades de soporte, ya que pasan a preocuparse también por los outputs que generan y tratan de mejorar constantemente su desempeño, ante la satisfacción de las necesidades de otras áreas de trabajo de la empresa.

Así, el estudio del caso de Tecnológica S.A. ha evidenciado que los instrumentos de control de gestión contribuyen en gran medida al éxito del negocio, mediante la difusión de los objetivos y de la estrategia, de alineación de las actividades de los distintos niveles jerárquicos y funcionales, pero también mediante el acceso a una información más detallada sobre el desarrollo de las áreas de negocio, lo que permite comprobar las fuentes de creación de valor de la actividad.

\section{Referencias}

Anthony, Robert \& Govindarajan, Vijay (2007). Management Control Systems. $12^{\mathrm{a}}$ ed. New York: McGraw-Hill.

Araújo, Cidália; Pinto, Emília M. F.; Lopes, José; Nogueira, Luís \& Pinto, Ricardo (2008). Estudo de caso. Mestrado em Educação, Área de Especialização em Tecnologia Educativa, Instituto de Educação e Psicologia, Universidade do Minho. Disponible en: http://grupo4te. com.sapo.pt/estudo_caso.pdf

Chiavenato, Idalberto (1993). Introdução à teoria geral da administração. $4^{a}$ ed. São Paulo: Makron Books do Brasil.

Fernandes, Amarildo da Cruz (2003). Scorecard Dinâmico: Integrando a dinâmica de sistemas com o Balanced Scorecard. XXIII Encontro Nacional de Engenharia de Produção, ENEGEP, Ouro Preto, Brasil. Disponible en: http://www.abepro.org.br/biblioteca/ ENEGEP2003_TR0702_0847.pdf Horngren, Charles T.; Datar, Srikant M. \& Foster, George (2012). Cost Accounting - A Managerial Emphasis. 14ª ed. Upper Saddle River, New Jersey: Prentice Hall.

Jordan, Hugues; Neves, João Carvalho das \& Rodrigues, José Azevedo (2002). O Controlo de gestão - ao serviço da estratégia e dos gestores. $8^{\mathrm{a}}$ ed. Lisboa: Áreas Editora.

Kaplan, Robert \& Norton, David (2004). Mapas estratégicos. $6^{\mathrm{a}}$ ed. Rio de Janeiro: Editora Campos.

Kluge, Jürgen; Stein, Wolfram \& Licht, Thomas (2002). Gestão do Conhecimento - Segundo um estudo da McKinsey \& Company. Lisboa: Principia.

Neves, João Carvalho das (2011). Avaliação e Gestão da Performance Estratégica da Empresa. $2^{\mathrm{a}}$ ed. Lisboa: Texto Editores.

Neves, João Carvalho das (2012). Análise e relato financeiro - Uma visão integrada de gestão. $5^{\mathrm{a}}$ ed. Lisboa: Texto Editores.

Stake, Robert (1995). The Art of Case Study Research. Thousand Oaks, California: Sage Publications Inc.

Yin, Robert K. (2009). Case Study Research: Design and Methods. $4^{\text {th }}$ ed. Thousand Oaks, California: Sage Publications Inc.

- Fecha de recepción: 1 de julio de 2014

- Fecha de aceptación: 30 de octubre de 2014

- Disponible en línea: 15 de diciembre de 2014 
LA CONTRIBUCIÓN DE LOS SISTEMAS DE CONTROL DE GESTIÓN PARA EL ÉXITO EMPRESARIAL / N. DELICADo / 881

\section{Para citar este artículo}

Teixeira, Nuno Miguel Delicado (2014). La contribución de los sistemas de control de gestión para el éxito empresarial [número especial: Contabilidad Gerencial]. Cuadernos de Contabilidad, 15 (39), 853881.

doi: 10.11144/Javeriana.cc15-39.cscg 
